\title{
TTR
}

Traduction, terminologie, re?daction

\section{La langue médicale : une langue de spécialité à emprunter le temps d'une traduction}

\section{Maurice Rouleau}

Volume 8, numéro 2, 2e semestre 1995

Technolectes et dictionnaires

URI : https://id.erudit.org/iderudit/037216ar

DOI : https://doi.org/10.7202/037216ar

Aller au sommaire du numéro

Éditeur(s)

Association canadienne de traductologie

ISSN

0835-8443 (imprimé)

1708-2188 (numérique)

Découvrir la revue

Citer cet article

Rouleau, M. (1995). La langue médicale : une langue de spécialité à emprunter le temps d'une traduction. TTR, 8(2), 29-49. https://doi.org/10.7202/037216ar

\section{Résumé de l'article}

La langue médicale : une langue de spécialité à emprunter le temps d'une traduction-Comme une langue de spécialité naît du besoin que ressentent les spécialistes de communiquer entre eux de façon concise et sans ambiguïté, elle est forcément réservée aux initiés, c'est-à-dire aux spécialistes. Pour maîtriser cette langue, le traducteur ne doit pas seulement connaître les termes; il lui faut savoir les enchaîner dans un discours cohérent qui reflète les usages du domaine. Les particularités d'usage concernent tantôt le terme : son évolution et l'utilité de son étymologie; les besoins qui ont présidé à sa création; l'utilisation particulière des adjectifs; tantôt l'articulation du terme en discours : la préposition à utiliser; l'emploi des modes verbaux, etc. L'apprentissage de cette langue s'apparente donc, à bien des égards, à l'apprentissage d'une langue seconde. Le traducteur spécialisé dans le domaine médical doit connaître la langue médicale s'il veut produire un texte idiomatique. Et à l'instar de tout spécialiste, il n'utilisera cette langue que dans l'exercice de sa profession.
Tous droits réservés (C) TTR: traduction, terminologie, rédaction — Les auteurs, 1995
Ce document est protégé par la loi sur le droit d'auteur. L'utilisation des services d'Érudit (y compris la reproduction) est assujettie à sa politique d'utilisation que vous pouvez consulter en ligne.

https://apropos.erudit.org/fr/usagers/politique-dutilisation/ 


\section{La langue médicale: une langue de spécialité à emprunter le temps d'une traduction}

\section{Maurice Rouleau}

Une langue de spécialité naît du besoin que ressentent les spécialistes de communiquer entre eux de façon concise et sans ambiguité. C'est donc la façon qu'ont de s'exprimer les gens qui travaillent dans un même domaine ou sous-domaine de l'activité humaine. Cette façon de dire est forcément limitée; elle l'est, de fait, doublement. D'abord, elle est réservée aux spécialistes du domaine - elle a été créée par eux et pour eux. Elle leur est à l'occasion empruntée (ou devrait l'être) par les traducteurs et, encore plus rarement, par des imitateurs (Perec, 1991). De plus, elle est réservée à l'exercice de la profession. Le spécialiste, dans la vie courante, s'exprime comme M. Tout-le-monde; sa langue de spécialité ne lui est alors d'aucune utilité.

Comme les autres spécialistes, les traducteurs possèdent une langue propre pour communiquer entre eux sur leur activité, un métalangage pour décrire la traduction. Mais, à la différence des autres spécialistes, les traducteurs sont des professionnels qui travaillent quotidiennement sur les langues spécialisées existantes, qu'il doit connaître et utiliser à bon escient. Pour que son travail soit bon, le traducteur doit produire un texte idiomatique, c'est-à-dire produire un texte qui ressemble, à s'y méprendre, à celui qu'écrirait un spécialiste. Il doit donc, pour ce faire, utiliser la langue de spécialité de l'autre. De plus, comme tout spécialiste, le traducteur n'utilisera cette langue de spécialité (qui n'est pas sienne) que dans l'exercice de sa profession; autrement dit, il l'empruntera le temps d'une traduction. 
Dans le cas qui nous intéresse, à savoir la traduction médicale, le traducteur doit donc connaître la langue qu'utilise le médecin et, s'il ne la connaît pas, l'apprendre. À l'instar des autres langues de spécialité, la langue médicale se caractérise par la présence de termes techniques. Le spécialiste ne trouvant pas toujours dans la langue générale tous les éléments dont il a besoin pour exercer sa profession, il a dû créer de nouveaux termes. Le traducteur doit connaître ou apprendre ce vocabulaire. Mais un cours de langue médicale, ce n'est pas un cours de lexicologie. Le terme est une étiquette qui sert à désigner une réalité, rien de plus. Si l'apprentissage de la langue médicale se limitait au vocabulaire, le recours à un dictionnaire ${ }^{1}$, qui est une compilation plus ou moins complète des termes de la discipline, suffirait. Tel n'est pas le cas. Le vocabulaire fait certes partie du code linguistique, mais il n'en est qu'un élément.

Connaître l'équivalent français du terme anglais ne permet pas de traduire correctement un texte. Connaître l'étiquette ne suffit pas; pour que les mots aient un sens, il faut pouvoir comprendre ce que l'étiquette dit de la réalité. Par ailleurs, connaître la notion ne permet pas d'écrire spontanément de façon idiomatique. On peut savoir, par exemple, comment fonctionne le cœur et ne pas savoir le dire à la manière du spécialiste. Autrement dit, un cours de langue médicale, ce n'est pas un cours de médecine. Il serait d'ailleurs utopique, en 45

1. Dans le but de simplifier la lecture du texte, les différents dictionnaires sont identifiés par les sigles suivants:

FLAMM, voir Kernbaum (1994); GARDE, voir Delamare (1992); PMANU, voir Manuila L. (1991); GMANU, voir Manuila A. (19701981); GLADS, voir Gladstone (1990); PROB-.., voir Robert (19..). Quant au nombre de dictionnaires à consulter, un seul ne peut suffire, car la qualité des définitions varie beaucoup d'un dictionnaire à l'autre. Comparons, par exemple, comment trois dictionnaires médicaux couramment utilisés définissent le terme néoplasme.

(FLAMM): «Processus de multiplication cellulaire anarchique échappant à l'homéostasie. On oppose les néoplasmes bénins et les néoplasmes malins ou cancers.)

(GARDE): «Tissu morbide qui résulte du processus néoplastique. Tumeur, qu'elle soit bénigne ou maligne (cancer).» (PMANU): «S. de tumeur (en parlant, le plus souvent, de tumeur cancéreuse).» 
heures, de faire d'un traducteur un médecin! (L'inverse est aussi vrai.) Pour bien situer le terme dans son contexte, le traducteur a intérêt à consulter un dictionnaire médical à contenu encyclopédique ${ }^{2}$.

Traduire un texte médical de façon idiomatique, ce n'est pas insérer des termes techniques dans une langue générale. Quiconque se limiterait à cette façon de procéder risquerait de se faire répliquer par un spécialiste, comme cela est déjà arrivé: "C'est bien, mais c'est littéraire. " Ce que le spécialiste voulait dire, c'était qu'il aurait, lui, dit la même chose, mais autrement.

Il ne suffit donc pas de connaître les termes; il ne suffit pas de comprendre les notions; il ne suffit pas d'insérer des termes techniques dans une langue générale. Il faut savoir les enchaîner dans un discours cohérent qui reflète les usages du domaine.

S'il veut arriver à articuler son texte à la manière du spécialiste, le traducteur doit fréquenter assidûment les textes médicaux. Alors, tout comme l'enfant apprend à parler une langue par mimétisme, le traducteur s'imprégnera de certaines façons de dire auxquelles il

2. Fort heureusement, ils ont tous plus ou moins cette caractéristique. La consultation de plus d'un dictionnaire s'impose ici également. Comparons l'entrée sympathico-mimétique des trois dictionnaires médicaux précités.

(PMANU): "Qui a des effets similaires (sic) à ceux que l'on obtient en stimulant le système nerveux sympathique.»

(GARDE): «Se dit d'une substance dont l'action imite celle du sympathique. V. alphastimulant et bêtastimulant [...].»

(FLAMM): «Se dit de toute substance naturelle ou médicamenteuse, reproduisant les effets de la stimulation des fibres post-ganglionnaires du système sympathique. Les médiateurs chimiques de ce système, adrénaline et lévartérénol (V.c.m.), en constituent le type. V. adrénergique, amphétamine, carbazochrome, clonidine, dopamine, éphédrine, éthylnoradré-naline, étiléfrine, hydroxyamphétamine, isoprénaline, isoxsu-prine, lévisoprénaline, méphentermine, métaraminol, méthoxa-mine, méthoxyphénamine, naphazoline, orciprénaline, phénylé-prine, phénylpropanolamine, pipérazine (camsylatede), pseudo-éphédrine,salbutamol,synéphrine, tétryzoline, tymazoline.» 
pourra recourir spontanément dans ses traductions. De plus, il doit être un bon observateur des faits de langue qui se distinguent de ceux rencontrés dans la langue générale. L'évaluation de la fréquence des écarts observés l'amènera à décider si telle particularité fait partie de l'idiolecte ou du technolecte du spécialiste.

Connaître les usages du domaine, c'est connaître les caractéristiques de la langue médicale. Mais décrire ces caractéristiques n'est pas une mince tâche, et c'est une tâche à laquelle peu de gens se sont intéressés (Hamburger, 1982; Sournia, 1974). Dans le cadre du présent article, nous présentons quelques particularités de cette langue. Nous nous attardons d'abord à trois aspects du terme: son évolution, sa création et sa composition. Pour finir, nous abordons quelques aspects de l'articulation du terme en discours. Ces différents aspects seront examinés avec, comme toile de fond, l'utilité des dictionnaires pour leur apprentissage.

\section{Le terme}

Le terme n'est peut-être qu'un des éléments du code, mais il a une très grande importance, car il sert à exprimer de façon concise une réalité qui peut exiger parfois plusieurs lignes. Le traducteur médical doit donc se familiariser avec les termes médicaux et surtout ne pas se faire piéger, car leur forme est parfois trompeuse. Il arrive que l'étymologie, grecque ou latine, ne soit pas d'un grand secours à cause de l'évolution du sens de ces termes.

\section{1. Évolution et étymologie}

La maîtrise du vocabulaire médical repose sur la connaissance des racines grecques et latines. L'étymologie est donc essentielle à l'apprentissage de ce vocabulaire. Le sens, par exemple, d'endométrite peut facilement être appréhendé à partir de ses éléments de formation d'origine grecque: le radical métr- (matrice), le préfixe endo (à l'intérieur) et le suffixe -ite (inflammation). Ce terme désigne une inflammation de la muqueuse utérine. Celui de mégacaryocyte peut aussi être facilement appréhendé: formé de méga (grand), de karyon (noyau) et de kytos (cellule), ce terme désigne une cellule à gros noyau 
qui se rencontre dans la moelle osseuse. Dans de tels cas, l'étymologie est très utile.

Il peut arriver, par contre, que l'étymologie soit mauvaise, que le mot soit mal construit. C'est le cas de mots se terminant par -phérèse, p. ex. leucophérèse, qui devrait être leucaphérèse, car les racines grecques correspondant au sens du terme sont leucos et aphérein et non pas phérein (aphérein signifie le contraire de phérein). Les dictionnaires médicaux commencent à rectifier l'entrée: le FLAMM précise qu'il "serait étymologiquement plus correct de dire leucaphérèse» et le GARDE renvoie le lecteur à leucaphérèse.

Il peut arriver également que l'étymologie soit douteuse. Le terme anachlorhydrie est donné dans les dictionnaires médicaux comme synonyme de achlorhydrie. Le GARDE va même jusqu'à préciser que le terme est formé dư préfixe ana- privatif!

Il peut même arriver que l'étymologie soit déroutante. S'il est vrai que radiographie et radiogramme appartiennent au même domaine et désignent la même réalité, il n'en est pas de même de myélographie et de myélogramme. Le premier désigne la radiographie de la moelle épinière après injection, dans l'espace sous-arachnoïdien, d'opacifiant ou d'air, alors que le second désigne la formule indiquant les proportions respectives des différents éléments cellulaires de la moelle osseuse.

Il peut enfin arriver que l'étymologie ne soit d'aucune utilité pour comprendre le sens d'un terme. Prenons l'exemple de mélancolie. Formé de deux éléments grecs (mélas, mélanos: noir; kholê: bile), ce terme décrivait un état irascible et dépressif attribué à un excès de bile noire - explication qui ne tient évidemment plus de nos jours. De toute évidence, un terme, au moment de sa création, répond aux besoins et aux idées du temps. Mais au fur et à mesure que les connaissances évoluent, les besoins changent, et l'utilisateur doit décider s'il garde le terme tout en lui donnant un nouveau sens ou s'il crée un nouveau 
terme. Dans le cas qui nous intéresse, les médecins ont décidé de garder le terme, même si, ce faisant, sa motivation ${ }^{3}$ disparaissait.

Il arrive qu'un terme change de sens avec les ans et que les différentes acceptions qu'il a eues figurent au dictionnaire. Mais tous les dictionnaires ${ }^{4}$ ne s'entendent pas sur la nécessité de les y consigner. Si l'information ne figure pas dans un dictionnaire, c'est que les rédacteurs ont jugé que la nouvelle acception était suffisamment bien établie pour ne pas allonger indûment l'entrée avec des considérations historiques. L'évolution de sens dont il est question se situe dans une perspective diachronique et elle est considérée comme normale. Par contre, quand un tel changement de sens est en voie de se produire (perspective diachronique), ce glissement de sens n'est pas toujours bien vu. Prenons par exemple le terme étiologie. Le FLAMM ajoute après la définition: "C'est par un abus de langage (nous soulignons) qu'on utilise parfois ce terme pour désigner la cause même de la maladie, et non pas la science qui étudie cette cause.» Le PMANU définit ainsi le terme: «Étude des causes des maladies et, abusivement (nous soulignons), ces causes elles-mêmes». Quant au GARDE, il préfère ignorer le changement de sens qu'impriment à ce terme les utilisateurs et il ne consigne que le sens premier: "Étude des causes des maladies.» Pourquoi faudrait-il considérer comme abusif un glissement de sens en voie de s'implanter et comme normal un glissement de sens consacré par l'usage? Pourquoi faudrait-il refuser à étiologie la possibilité de désigner à la fois la science et l'objet de l'étude, alors qu'on l'accepte pour bien d'autres termes de même formation: étymologie, morphologie,

3. Au sens donné par le Petit Robert: «caractère d'un signe complexe dont le sens se déduit de ses composants».

4. Dans le GARDE, il est possible de savoir ce que le terme métastase signifiait: « $1^{\circ}$ Pour les Anciens...; $2^{\circ}$ Autrefois...; Actuellement...»Par contre dans le FLAMM et le PMANU, rien n'est consigné de façon aussi précise. Des considérations historiques sont parfois utiles aux traducteurs, surtout si le terme anglais n'a pas évolué au même rythme en anglais, comme cela est le cas pour metastasis. 
physiologie, terminologie 5 . Quant à «phraséologie», il semble n'avoir jamais signifié autre chose que "ensemble des expressions, locutions...» (PROBB-93), même s'il est formé, lui aussi, du suffixe -logie.

\section{Création d'un terme}

Les locuteurs créent un terme quand ils cherchent un moyen économique de dire la réalité. C'est ainsi que le spécialiste a créé inhibiteur calcique pour désigner un composé qui inhibe le passage à travers la membrane cellulaire des ions calcium grâce à des unités fonctionnelles, les canaux calciques. L'économie que représente inhibiteur calcique est plus qu'évident.

Il est un terme qui a été créé par le spécialiste et qui suscite beaucoup de réticence de la part des langagiers. C'est le terme "greffé». Le médecin qui effectue une greffe d'organe ne va pas, chaque fois qu'il veut parler du type de patient qu'il traite, utiliser «receveur de greffe». Cela est trop long. Il a opté pour greffé ou transplanté. Une analyse serrée de ce substantif fait dire aux puristes, ou défenseurs de la langue française, que c'est l'organe qui est greffé et non le patient; par conséquent, utiliser greffé pour désigner le sujet qui a reçu une greffe est contraire au bon sens. Le médecin, lui, ne se formalise pas de cet accroc à la logique. Il a eu recours à greffé parce qu'il connaissait d'autres termes, fort utiles, appartenant aussi bien à la langue médicale qu'à la langue générale, qui sont de même construction: ménopausée, handicapé, miraculé, accidenté, médaillé, gazé, etc. C'est le modèle qu'inconsciemment sans doute il a suivi. Le médecin serait en droit de se demander pourquoi lui ne pourrait pas recourir à ce procédé pour former un mot, alors qu'en langue générale ce procédé est déjà utilisé. Il est bien évident qu'au moment de la création de ce terme le spécialiste n'y a vu que le côté pratique. Ce terme pose peut-être un

5. Étymologie: $1^{\circ}$ Science de la filiation des mots...; $2^{\circ}$ origine ou filiation d'un mot.

Morphologie: $1^{\circ}$ Étude de la configuration...; $2^{\circ}$ forme, configuration. Physiologie: Science qui étudie le fonctionnement et les propriétés des organes...; par ext. fonctionnement.

Terminologie: $1^{\circ}$ Vocabulaire particulier utilisée dans un domaine de la connaissance; $2^{\circ}$ Étude systématique des termes... 
problème au langagier, mais il n'en pose aucun au spécialiste. Ce dernier sait très bien ce qu'il veut dire quand il parle d'un greffé.

\section{Composition des termes médicaux}

Le sens des termes médicaux, nous venons de le voir, n'est pas toujours évident; l'étymologie, qui devrait aider à comprendre le sens, pose des difficultés que le traducteur se doit de connaître. La solution à ces difficultés se trouve, assez souvent, dans les dictionnaires spécialisés.

Il est un autre aspect du terme médical qui peut présenter des pièges, c'est la construction des termes formés d'un substantif + un ou plusieurs adjectifs. Les adjectifs en question peuvent tout aussi bien être des adjectifs de relation (ex.: infarctus pulmonaire), des adjectifs qualificatifs (ex.: abdomen aigu) que les deux à la fois (leucémie lymphoïde chronique). Examinons donc quelques problèmes soulevés par de tels adjectifs quand ils entrent dans la formation d'un terme à un seul adjectif ou à plusieurs adjectifs.

\section{A. Terme à un seul adjectif}

Avec les termes à un seul adjectif, deux problèmes retiendront notre attention: 1. l'interchangeabilité de l'adjectif de relation et du groupe prépositionnel correspondant; 2. la construction de l'adjectif en hypallage.

\section{Adjectif de relation / Groupe prépositionnel (GP)}

Un des traits caractéristiques de l'adjectif de relation, c'est qu'il s'interprète comme un groupe, ou syntagme, prépositionnel (Maingueneau, 1994, p. 52): élection présidentielle et élection du président. Dans ce cas-ci, les deux se disent, ils sont interchangeables.

En langue médicale, il existe beaucoup de termes qui acceptent aussi bien l'adjectif de relation que le GP. Ce sont des termes non figés. En voici deux exemples: contraction ventriculaire / du ventricule, numération plaquettaire / des plaquettes. Il en existe d'autres, par contre, où l'adjectif de relation et le GP ne sont pas interchangeables. Cette non-interchangeabilité ne tient pas à une variation de sens, mais bien à 
l'usage. Personne n'aurait l'idée de rendre angina pectoris par «angine pectorale», même si l'adjectif «pectoral» signifie «de la poitrine» et que «région pectorale» se dit. Il en est de même pour l'adjectif «myocardique» (qui se rapporte au myocarde). Tonus myocardique et irrigation myocardique, par exemple, s'utilisent aussi bien que tonus du myocarde et irrigation du myocarde. Il serait donc bien tentant de traduire myocardial infarction par «infarctus myocardique», d'autant plus que le terme anglais est formé lui-même d'un adjectif (myocardial). Mais ce faisant, le traducteur se trahirait. Il afficherait son ignorance de la langue médicale. Ce n'est pas que «infarctus myocardique» et «infarctus du myocarde» aient des sens différents, c'est simplement que le médecin n'a pas l'habitude de parler d'un «infarctus myocardique».

Ce qui est vrai de myocardial infarction ne l'est pas de pulmonary infarction. Ce dernier se traduit par «infarctus pulmonaire» et non par «infarctus du poumon». Par ailleurs, intestinal infarction se rend aussi bien par «infarctus mésentérique», par «infarctus entéromésentérique» que par «infarctus de l'intestin» (GMANU). Comme ces termes français figurent dans les dictionnaires médicaux, il est inexcusable qu'un traducteur se laisse piéger. En fait, si l'association d'un substantif et d'un groupe prépositionnel forme un terme figé, il est fort probable qu'il se trouvera consigné dans un dictionnaire médical.

\section{Adjectif construit en hypallage}

Il y a hypallage lorsqu'est attribué à un mot ce qui, dans les faits, convient à un autre mot qui, lui, est absent.

Adjectif construit normalement Adjectif construit en hypallage

1. système veineux

2. solution sucrée

3. infection hépatique

4. infection vaginale
5. stase veineuse

6. diabète sucré

7. coma hépatique

8. hystérectomie vaginale

Il est clair que l'adjectif, en 1, 2, 3 et 4, se rapporte directement au substantif qui le précède: le système des veines, la solution de sucre, 
l'infection du foie et l'infection vaginale. En 5, 6, 7 et 8, la situation est tout autre. Il ne s'agit pas d'une stase de la veine, mais d'une stase du sang veineux; il ne s'agit pas du diabète du sucre, mais du diabète aux urines sucrées; il ne s'agit pas d'un coma du foie, mais d'un coma secondaire à une atteinte du foie; enfin, il ne s'agit pas d'un hystérectomie du vagin, mais d'une hystérectomie par voie vaginale (sont soulignés les mots absents qui sont qualifiés par l'adjectif en hypallage).

Par souci d'efficacité - l'efficacité passe forcément par la concision -, le spécialiste évite de dire ce qui est évident à lui et à tous ceux à qui il s'adresse. Ses énoncés ont donc un caractère elliptique. Ces formes ramassées ne plaisent pas au non-spécialiste qu'est le traducteur; il les juge mal construites parce qu'il n'en saisit pas d'emblée le sens. Ce reproche n'est pas justifié, car ce n'est pas sa langue, mais bien celle des spécialistes. Certains vont même jusqu'à considérer qu'un étoffement s'impose pour bien expliciter la notion. Une telle recommandation n'est pas plus justifiée. Il y aurait peut-être lieu de rappeler au non-spécialiste deux vérités: 1 . Moins on en connaît, plus on en met $^{6}$. Et cette loi s'applique fort bien ici: moins le traducteur connaît la langue médicale, plus sa traduction sera longue. Il est donc normal que le médecin qui en connaît beaucoup et qui s'adresse à des gens qui, eux aussi, en connaissent autant utilise peu de mots (souci d'économie). Le traducteur qui doit lui emprunter sa langue le temps d'une traduction s'il veut produire un texte idiomatique n'a pas le droit d'en mettre plus. 2. Ne jamais reprocher aux autres ce que l'on fait soi-même. Si le non-spécialiste juge qu'il vaudrait mieux abolir ces constructions en hypallage sous prétexte qu'elles sont incompréhensibles à la première lecture, il se devrait, pour être conséquent avec lui-même, faire disparaître de sa langue générale des termes qui pèchent par le même défaut: finale masculine de Wimbledon, place assise, zone franche, guerre sanglante, blessé grave ${ }^{7}$, etc. Ces termes représentent un

6. C'est la «loi de Buisseret»: moins un traducteur connaît le français, plus sa traduction est prolixe. (Buisseret, I. de, 1975, p. 9)

7. Dans le PROB-77 et le PROB-92, «blessé grave» est consigné. Ce n'est que dans le PROB-93 que son emploi est critiqué. Un sursaut de purisme après tant d'années a de quoi étonner. Normalement c'est 
obstacle à la compréhension pour qui veut apprendre le français, tout comme les termes médicaux en représentent un à qui veut lire un texte médical et le comprendre. Il y aurait peut-être lieu de se rappeler qu'apprendre une langue de spécialité, c'est, à bien des égards, apprendre une langue «étrangère».

Le médecin fut sans doute, à une certaine époque, sensible au caractère hermétique des termes formés d'un adjectif construit en hypallage, car il a créé quelques nouveaux adjectifs (coronarien, carotidien, tricuspidien, gravidique, etc.) qui viennent rétablir le rapport normal avec les substantifs qui les accompagnent. Prenons le cas de coronaire / coronarien. Dans «insuffisance coronaire», le mot absent que l'adjectif qualifie est "vaisseau». En effet, coronaire se dit «des vaisseaux disposés en couronne», d'où artère coronaire. La création de "coronarien» (qui se rapporte aux vaisseaux coronaires) permet de construire un terme (insuffisance coronarienne) qui, cette fois, est explicite, car il désigne l'insuffisance des vaisseaux coronaires. Mais ceux-là même qui avaient senti le besoin d'un adjectif autre que coronaire pour qualifier une insuffisance, c'est-à-dire les médecins, ont depuis changé d'idée. Cet adjectif, après presque un siècle d'existence, ne semble plus avoir sa raison d'être. En effet, le Comité d'Études des termes médicaux français a jugé, en 1987, que «insuffisance coronaire» était préférable à «insuffisance coronarienne», mais que les deux étaient utilisables $^{8}$. Le traducteur peut trouver cette paire d'adjectifs fort utile, mais les médecins, eux, ne semblent plus du même avis!

\section{B. Terme à plus d'un adjectif}

Le problème soulevé par des syntagmes formés d'au moins deux adjectifs porte sur l'ordre de ces derniers dans le syntagme. Personne n'oserait dire une «colonne solide vertébrale». La place obligée de l'adjectif «solide» dans ce syntagme s'expliquerait par le fait que

l'inverse qui se produit: las de condamner une tournure, le puriste, sous la pression de l'usage, finit par l'accepter (ex. «approche» au sens de "manière d'aborder un sujet de connaissance...»).

8. Extrait du procès-verbal de la séance du 19 février 1987. Cité dans l'Actualité terminologique, XXII(1), 1989, p. 7. 
"colonne vertébrale» forme un syntagme figé. Une telle explication est certes valable, mais elle n'est pas d'une très grande utilité quand il n'y a pas de syntagme figé dans le terme. Doit-on dire, par exemple, "concentration minimale inhibitrice» ou «concentration inhibitrice minimale»? Le principe qui régit l'ordre des adjectifs veut qu'en français on détermine avant de qualifier. C'est dire que le premier adjectif concerne la nature du substantif (qualité essentielle) et le second, un attribut variable (qualité accessoire). Il faudrait donc, ici, dire une "concentration inhibitrice minimale», ce qui correspond d'ailleurs parfaitement à la composition du syntagme anglais (Attal, 1987, p. 133) minimum inhibitory concentration. Dans le FLAMM, on trouve l'inverse: "concentration minimale inhibitrice». Même si grammaticalement le traducteur aurait raison d'écrire «concentration inhibitrice minimale», il doit se conformer à l'usage ${ }^{9}$ et utiliser «concentration minimale inhibitrice». Et ce cas n'est pas unique.

Examinons la place des adjectifs «chronique» et «aigu» dans des termes formés de plus d'un adjectif. Le médecin parle toujours d'une insuffisance rénale aiguë ou chronique, d'un lupus érythémateux aigu ou chronique, ou encore d'un cœur pulmonaire aigu ou chronique. Les adjectifs «aigu» et "chronique» viennent toujours en seconde place, car ils désignent une qualité accessoire. Fort d'une pratique qui semble bien établie et bien justifiée, le traducteur est donc amené à traduire chronic lymphocytic leukemia par «leucémie lymphoïde chronique», et il a raison. Par contre, il n'aurait pas raison de rendre acute lymphocytic leukemia par leucémie lymphoblastique (ou lymphoïde) aiguë. Il lui aurait fallu écrire leucémie aiguë lymphoblastique (ou lymphoïde). Tel est l'usage, et cet usage est consigné dans les dictionnaires médicaux français.

Le problème se pose également quand le substantif est suivi de trois adjectifs. Doit-on traduire diffuse interstitial pulmonary fibrosis par «fibrose pulmonaire interstitielle diffuse» (GARDE) ou par «fibrose interstitielle diffuse pulmonaire» (FLAMM)? Comment traduire left

9. Comme ce terme ne figure que dans un dictionnaire, la notion d'usage pourrait être contestée. Il faut toutefois se rappeler qu'un dictionnaire reflète, mais toujours en retard, l'usage que font d'un terme les utilisateurs. 
ventricular end diastolic pressure (LVEDP)? Par «pression télédiastolique ventriculaire gauche», comme le propose le GLADS ou par «pression ventriculaire gauche télédiastolique»? LVEDP désigne la pression qui existe dans le ventricule gauche (ventriculaire gauche) à la fin de la diastole (télédiastolique). Étant donné que «pression» et «du ventricule gauche» ne forment pas un syntagme figé, il est possible d'intercaler l'adjectif «télédiastolique» entre les deux éléments. On peut donc dire «pression télédiastolique du ventricule gauche» ou «pression du ventricule gauche diastolique» (la préférence semble toutefois aller à la première forme). Cependant, quand on remplace le groupe prépositionnel (du ventricule gauche) par l'adjectif correspondant (ventriculaire gauche), il faudrait sans doute permuter les adjectifs, car, en français, on détermine avant de qualifier. Cependant, l'usage est-il suffisamment bien établi, chez les utilisateurs de ces termes, pour que le traducteur se sente contraint d'utiliser une forme plutôt que l'autre, s'il veut que son texte soit le plus idiomatique possible? Seule la lecture de documents médicaux écrits par de nombreux auteurs différents permettrait de trancher. Les termes à trois adjectifs dont il vient d'être question ne semblent pas constituer, aux yeux des rédacteurs de dictionnaires, des termes figés. En effet, dans le premier cas, deux dictionnaires (FLAMM et GARDE) utilisent des termes différents pour désigner la même réalité; dans le second, le terme ne se trouve dans aucun dictionnaire médical unilingue français; seul le GLADS le mentionne.

\section{L'articulation du vocabulaire en discours}

Nous avons mentionné précédemment qu'il ne fallait pas confondre langue de spécialité et vocabulaire de spécialité et que la langue ne se résume pas au vocabulaire. Une langue de spécialité, c'est la façon de communiquer que partagent les spécialistes d'un domaine. Autrement dit, pour qui veut l'apprendre, il lui faut non seulement connaître les termes techniques, mais savoir les enchaîner dans un discours cohérent qui reflète les usages du domaine.

L'articulation des termes en discours est assurément l'aspect de la langue de spécialité qui est le moins étudié, le moins facile à maîtriser. Cette difficulté tient au fait que les façons de dire ne sont pas 
consignées comme telles dans les dictionnaires médicaux et que, de plus, l'évolution du sens des termes peut amener un changement dans les cooccurrents à utiliser.

Prenons le cas du terme "posologie» (équivalent du terme anglais "dosage»). Traduire In the case of renal failure, the dosage should be reduced peut sembler facile à première vue. En fait, traduire cette courte phrase présente une difficulté qui tient au sens du verbe reduced. L'équivalent qui vient immédiatement à l'esprit, à savoir «réduire», n'est utilisable que si la réalité désignée par dosage est réductible. La solution se trouve donc dans le sens du terme "posologie». Si ce dernier désigne la «quantité totale d'un médicament estimée selon l'âge...» (PMANU), le verbe "réduire» convient, car une quantité peut être réduite. $\mathrm{Si}$, par contre, ce terme désigne l'«indication figurant sur une préparation spécialisée...» (FLAMM), il est clair que l'indication ne peut être réduite; elle peut cependant être modifiée. Le choix du cooccurrent dépend donc du sens donné au terme, et ce sens dépend, à son tour, du dictionnaire consulté ${ }^{10}$.

Prenons maintenant le cas du substantif «traitement» ou du verbe «traiter», mots qui n'appartiennent pas exclusivement au domaine médical. Faut-il traduire he has been treated with steroids par «il a été traité par des stéroïdes, avec des stéroïdes ou aux stéroïdes»? Le problème qui se pose ici est celui de l'expression de l'instrumentalité, du moyen. En français, les prépositions par, avec et à servent toutes trois à exprimer le moyen: obtenir par la force, manger avec les doigts, observer à l'œil nu. Le traducteur médical pourrait croire que les trois prépositions peuvent être utilisées indifféremment. La question qu'il devrait se poser n'est pas de savoir si les trois peuvent se dire, mais bien plutôt de savoir laquelle des trois se dit en pratique. Pour que sa traduction soit idiomatique, le traducteur médical doit utiliser par (Rouleau, 1994, p. 199). Ce qui est vrai de la langue médicale ne l'est pas obligatoirement des autres langues de spécialité. "Traiter», nous

10. Les différents dictionnaires médicaux ne reflètent pas nécessairement tous l'usage. Certains se veulent plus normatifs que descriptifs. Le GARDE, par exemple, ne donne, à «posologie», que son sens étymologique: étude des doses thérapeutiques des divers médicaments suivant l'âge, le sexe et l'état du malade. 
l'avons dit, n'est pas un verbe réservé au domaine médical, il fait également partie, par exemple, du vocabulaire de l'ingénieur métallurgiste, et rien ne dit que ce spécialiste ${ }^{11}$ a recours à la même préposition que le médecin. Et l'agronome, lui, veut-il qu'un champ soit traité au D.D.T. (PROB-93)? Seule la lecture de documents traitant du domaine en question permettrait de connaître la façon de dire des spécialistes du domaine.

À la lecture d'un article sur les antihelminthiques (Bourée, 1982), il est possible de remarquer que l'auteur utilise la préposition sur quand l'adjectif «actif» est suivi du nom du microorganisme responsable de l'affection (actif sur les douves), et de préférencela préposition dans quand l'adjectif est suivi du nom de la maladie (actif dans la bilharziose). La préférence notée chez cet auteur se retrouve-t-elle chez d'autres? Si oui, le traducteur aurait intérêt à utiliser les mêmes prépositions dans les mêmes circonstances, s'il veut produire un texte idiomatique. L'examen des prépositions utilisées par le même auteur après l'adjectif «efficace» révèle par contre une variabilité: «efficace dans les bilharzioses», "efficace sur les bilharzioses» et "efficace contre telle maladie». Quelle est la solution? Il n'en existe peut-être pas. Et s'il en existait une, elle ne se trouverait pas nécessairement dans le PROB, car ce dernier fait appel, pour ses exemples, à des œuvres littéraires. On trouve, par exemple, à «efficace» une citation de Jules Romains: «telle eau est efficace pour les dermatoses» (Romains). Que J. Romains utilise pour ne signifie pas que le médecin utilise la même préposition. Le traducteur doit donc faire la distinction entre ce que fournit un dictionnaire général et les exigences, non consignées, de la langue de spécialité. Autrement dit, ce n'est pas dans un dictionnaire de

11. Le problème de la préposition n'est pas particulier à la langue médicale. Il se rencontre également en traduction technique (Durieux, 1988): Welding by laser, cutting by laser, drilling by laser n' appellent pas obligatoirement la même préposition. Il est courant de dire «soudure par laser, découpe $a u$ laser et perçage par laser». 
langue générale qu'un traducteur spécialisé trouvera les habitudes langagières du spécialiste du domaine ${ }^{12}$.

Jetons maintenant un rapide coup d'œil sur l'emploi des modes verbaux. La langue médicale, tout comme la langue juridique ${ }^{13}$ d'ailleurs, n'utilise pas le on (Rouleau, 1993). Pourtant ce pronom indéfini pullule dans les textes médicaux traduits. Ce phénomène traductionnel s'explique sans doute par l'anathème jeté par les professeurs de traduction sur la forme du passif. Ils ont interprété comme un interdit la caractéristique relevée par Vinay et Darbelnet (1958) concernant l'emploi du passif et de l'actif en français et en anglais. Pourtant, Darbelnet a nuancé sa position ${ }^{14}$, mais on n'en a pas pris note. La fréquence du passif n'est pas condamnable; ce n'est pas, comme certains veulent bien le croire, un calque de l'anglais. Il s'agit d'une façon de dire qui répond à deux besoins réels: mettre l'accent sur la chose étudiée et taire l'identité de l'observateur. Mettre comme sujet de la phrase l'objet de l'étude est tout à fait normal; c'est ce dont l'auteur veut nous parler. Cette façon de dire n'empêche pourtant pas l'auteur de l'article de préciser qui est l'agent (p. ex. Ce phénomène a été observé par nous), mais il ne le fait pas. L'auteur est un scripteur anonyme; il s'efface derrière l'objet de son discours, car le fait rapporté ne tient pas à SA participation: tout autre chercheur aurait, dans les mêmes conditions, fait la même observation.

12. Dans PROB-93, «réprimer», au sens de «arrêter l'effet, l'action de» est dit $v x$. méd. Le médecin ne dirait-il pas d'une antiémétique qu'il réprime les vomissements? Si oui, serait-ce un hapax?

13. «La langue juridique ne dit jamais on (alors que celle des guides de tourisme y a constamment recours. Cette absence du pronom indéfini entraîne naturellement par compensation un emploi assez fréquent du passif.» (Darbelnet, 1982, p. 58)

14. «Il convient de se débarrasser du préjugé selon lequel le passif est à éviter en français, sans doute parce qu'il apparait comme un reflet de l'anglais. En fait le passif est de mise chaque fois que le sujet subit l'action et que l'agent de cette action reste au second plan ou même est purement et simplement omis: "La question a été réglée hier".» (Darbelnet, 1982, p. 58) 
Ces quelques considérations offrent, à celui qui veut étudier en détail une langue de spécialité, tout le domaine des cooccurrents: comment le médecin parle-t-il des signes et des symptômes, du diagnostic, du pronostic, etc.? Quels sont les verbes, les adjectifs associés à ces termes dans son discours? Selon le médecin, de quoi le patient peut-il être porteur? Un être peut-il souffrir d'une maladie qui ne s'accompagne pas de douleur, comme l'hypertension? Voilà autant de questions, qui concernent l'articulation en discours, auxquelles le traducteur doit trouver réponse pour produire un texte idiomatique. Ce vaste domaine de la phraséologie a trop longtemps été négligé par les terminologues. Et ceux qui disent étudier les langues de spécialité ${ }^{i 5}$ ont peut-être encore trop tendance à confondre vocabulaire de spécialité et langue de spécialité: la terminologie n'est pas une langue.

Voilà brossés à très grands traits ce qu'est la langue médicale et ce que doit être l'attitude du traducteur à son égard. La langue médicale est une langue de spécialité parce qu'elle a été créée par des spécialistes pour des spécialistes. Elle appartient donc au médecin et non au traducteur qui peut, certes, la lui emprunter le temps d'une traduction, mais assurément pas se l'approprier.

À l'instar de toutes les langues de spécialité, la langue médicale se caractérise, entre autres, par la présence de termes techniques. Ces termes sont facilement reconnaissables parce que leur forme est généralement rebutante pour le profane ou le non-spécialiste (dont fait partie le traducteur) ${ }^{16}$. Pour saisir le sens de ces termes, l'étymologie est en général d'un très grand secours, parfois d'aucune aide. Ces termes

15. "Chaque discipline, chaque branche de la connaissance a sa propre terminologie. Le droit ne fait pas exception à cette règle. La terminologie juridique est une langue technique à l'intérieur de la langue usuelle.» (Kerby, 1982, p. 5)

16. Il existe une autre catégorie de termes techniques dont il n'a pas été question ici: les mots ordinaires (aux formes non rebutantes) qui ne sont des termes techniques que par un de leurs sens (succulent $=$ œedématié; exonération = défécation). Le sens de ces termes peut ou non être consigné dans un dictionnaire de langue. Il ne l'est pas nécessairement dans un dictionnaire de spécialité. 
se retrouvent normalement dans les dictionnaires spécialisés. Comme ces derniers ne sont pas tous d'égale valeur, il est bon que le traducteur en consulte plus d'un. Et son attention devrait se porter en particulier sur ceux qui présentent un caractère encyclopédique. C'est dans ceux-ci qu'il pourra le mieux situer le terme dans son contexte normal d'utilisation, ce contexte lui étant d'autant plus nécessaire qu'il n'est pas spécialiste en la matière.

La langue médicale ne se caractérise cependant pas uniquement par son vocabulaire technique. Elle se caractérise également, comme nous l'avons illustré, par l'articulation du vocabulaire en discours. Pour exprimer ses réalités, le médecin utilise des tournures particulières (par exemple, traiter par), tournures qui ne figurent ni dans les dictionnaires spécialisésni dans les dictionnaires généraux; elles ne se rencontrent que dans les textes écrits par ces spécialistes.

Pour connaître la façon de dire des médecins, le traducteur doit donc lire beaucoup d'ouvrages médicaux et être très attentif aux faits de langue qui se distinguent de ceux que contient la langue générale. Ce n'est qu'à ce prix que le traducteur médical pourra produire une bonne traduction, un texte dont on ne saurait dire s'il est une traduction ou non. Il ne faut jamais oublier que, aujourd'hui, apprendre une langue de spécialité, c'est un peu comme apprendre une langue étrangère... à l'oreille. Un jour, peut-être sera-t-il possible de l'apprendre d'une autre manière? À la condition toutefois qu'on étudie cette langue pour ce qu'elle est, c'est-à-dire une langue de spécialité, et non pour ce qu'on voudrait qu'elle soit, une langue générale farcie de termes techniques.

Maurice Rouleau: Département de langues modernes et de traduction, Université du Québec à Trois-Rivières, CP 500, TroisRivières (Qc) G9A 5 H7.

Courrier élec.: Maurice_Rouleau@uqtr.uquebec.can 


\section{Références}

ATTAL, Jean-Pierre (1987). Grammaire et usage de l'anglais. ParisGembloux, Duculot.

BOURÉE, Patrice (1982). «Antihelminthiques». Encycl Méd chir, Paris, Thérapeutique, $25061 \mathrm{~A}^{10}, 4-1982$.

BUISSERET, Irène de (1975). Deux langues, six idiomes. Ottawa, Carlton-Green.

DARBELNET, Jean (1982). «Niveaux et réalisations du discours juridique». Langage du droit et traduction. J.-C. Gémar, dir. (1982).

DELAMARE, Jacques(1992). Dictionnaire des termes de médecine. $23^{\mathrm{e}}$ édition, Paris, Maloine.

DURIEUX, Christiane (1988). Fondement didactique de la traduction juridique. Paris, Didier Érudition.

GÉMAR, J.-C., dir. (1982). Langage du droit et traduction. Brossard, Linguatech.

GLADSTONE W.J. (1990) Dictionnaire anglais-français des sciences médicales et paramédicales. $3^{\mathrm{e}}$ édition, St-Hyacinthe, Edisem.

HAMBURGER, Jean (1982). Introduction au langage de la médecine. Paris, Flammarion Médecine-Sciences.

KERBY, Jean (1982). "La traduction juridique, un cas d'espèce». Langage du droit et traduction. J.-C. Gémar, dir. (1982).

KERNBAUM, Serge (1994). Dictionnaire de médecine Flammarion. $5^{\mathrm{e}}$ édition, Paris, Flammarion Médecine-Sciences.

MAINGUENEAU, Dominique (1994). Précis de grammaire pour les concours. Paris, Dunod. 
MANUILA, A. et al. (1970-1981). Dictionnaire français de médecine et de biologie. Paris, Masson.

MANUILA, L. et al. (1991). Dictionnaire médical. $4^{\mathrm{e}}$ édition. Paris, Masson.

PEREC, Georges (1991). Cantatrix sopranica L. et autres écrits scientifiques. Paris, Seuil.

ROBERT, Paul (1977). Le petit Robert 1. Paris, Le Robert.

(1992). Le petit Robert 1. Paris, Le Robert.

(1993). Le nouveau petit Robert. Paris, Dictionnaire Le

Robert.

ROULEAU, Maurice (1993). «La voix passive dans les textes médicauX et paramédicaux). Meta XXXVIII(3), pp. 440-448.

(1994). La traduction médicale. Une approche méthodique. Brossard, Linguatech.

SOURNIA, J.-C. (1974). Langage médical moderne. Paris, Hachette.

VINAY, J.-P. et J. Darbelnet (1958). Stylistique comparée du français et de l'anglais. Montréal, Beauchemin.

RÉSUMÉ: La langue médicale: une langue de spécialitẻ à emprunter le temps d'une traduction - Comme une langue de spécialité naît du besoin que ressentent les spécialistes de communiquer entre eux de façon concise et sans ambiguité, elle est forcément réservée aux initiés, c'est-à-dire aux spécialistes. Pour maîtriser cette langue, le traducteur ne doit pas seulement connaître les termes; il lui faut savoir les enchaîner dans un discours cohérent qui reflète les usages du domaine. Les particularités d'usage concernent tantôt le terme: son évolution et l'utilité de son étymologie; les besoins qui ont présidé à sa création; l'utilisation particulière des adjectifs; tantôt l'articulation du terme en discours: la préposition à utiliser; l'emploi des modes verbaux, etc. L'apprentissage de cette langue s'apparente donc, à bien des égards, 
à l'apprentissage d'une langue seconde. Le traducteur spécialisé dans le domaine médical doit connaître la langue médicale s'il veut produire un texte idiomatique. Et à l'instar de tout spécialiste, il n'utilisera cette langue que dans l'exercice de sa profession.

ABSTRACT: Medical Language: Switching into LSP when Translating - Since an LSP develops out of the perceived need of specialists to intercommunicate in a precise and non-ambiguous manner, the use of such a language will necessarily be restricted to insiders, i.e. specialists. It is not sufficient that the translator be familiar with the terms and expressions of a given LSP; he or she must also be able to incorporate them into a coherent discourse that faithfully reflects the usages peculiar to that field of specialization. These particularities of use embrace the terms employed: the way they have developed and the relevance of their etymology; the needs that impelled the creation of these terms; the specific use of adjectives. The particularities of use also extend to the discursive framework: the choice of preposition, the use of tenses, and so on. Learning an LSP is, thus, in many respects similar to learning a second language. Translators who specialize in the field of medicine must be familiar with medical language if they wish to produce an idiomatic text. However, like all specialists, they will only use this language in the exercise of their profession; once the translation is finished, they will switch back to normal discourse. 